Archives of Biochemistry and Btophysics

Vol. 189, No. 2, August, pp. 508-515, 1978

\title{
Evidence for Two Sites of Inhibition of Photosynthetic Electron Transport by Dibromothymoquinone
}

\author{
JAMES A. GUIKEMA AND CHARLES F. YOCUM ${ }^{1}$ \\ Department of Cellular and Molecular Biology, University of Michigan, Ann Arbor, Michigan 48109
}

Received November 30, 1977; revised March 15, 1978

\begin{abstract}
Two sites in the photosynthetic electron transport chain of spinach chloroplasts are sensitive to inhibition by the plastoquinone antagonist dibromothymoquinone (2,5-dibromo3-methyl-6-isopropyl-p-benzoquinone). This compound imposes maximal inhibition on reactions involving electron transport from water to a terminal acceptor such as ferricyanide at concentrations of about $1 \mu \mathrm{M}$. At concentrations of about $10 \mu \mathrm{M}$, dibromothymoquinone also inhibits electron transport reactions catalyzed by photosystem II in the presence of $p$ phenylenediimines or $p$-benzoquinones. This inhibition is observed in both untreated and KCN/Hg-inhibited chloroplast preparations. Thiol incubation of chloroplasts exposed to dibromothymoquinone relieves inhibition at both sites. This reversal of inhibition is, however, different for the two sites. Restoration of ferricyanide reduction, which is blocked by $1 \mu \mathrm{M}$ dibromothymoquinone, required high thiol/inhibitor ratios and incubation times with thiol of up to $3 \mathrm{~min}$. The reversal of inhibition of $p$-phenylenediimine reduction by photosystem II, on the other hand, requires a thiol/inhibitor ratio of 1, and incubation times as short as $5 \mathrm{~s}$. Addition of bovine serum albumin to absorb dibromothymoquinone results in a partial restoration of photosystem II reactions, but ferricyanide reduction, which requires photosystem II and photosystem I, cannot be restored by this procedure.
\end{abstract}

Dibromothymoquinone (DBMIB), ${ }^{2}$ a well established inhibitor for use in studies of photosynthetic electron transport, has been shown to inhibit the flow of electrons between photosystems (PS) II and I, presumably by interference with plastoquinone function (1). Noncyclic electron transport from water to ferricyanide is inhibited more than $60 \%$ by DBMIB concentrations of approximately $1 \mu \mathrm{M}$. Photosystem II-dependent noncyclic electron transport can be restored in DBMIB-poisoned chloro-

\footnotetext{
${ }^{1}$ To whom correspondence should be addressed.

${ }^{2}$ Abbreviations used are: DBMIB, 2,5-dibromo-3methyl-6-isopropyl-p-benzoquinone (dibromothymoquinone); Chl, Chlorophyll; DAD, 2,3,5,6-tetramethyl$p$-phenylenediamine; DAT, 2,5-diaminotoluene; DCBQ, 2,6-dichloro-p-benzoquinone; DMQ, 2,5-dimethyl-p-benzoquinone; DPIP, 2,6-dichlorophenol-indophenol; DTT, dithiothreitol; PD, $p$-phenylenediamine; PS I, photosystem I; PS II, photosystem II; TMPD, tetramethyl- $p$-phenylenediamine. The subscript -ox following abbreviations for $p$-phenylenediamine electron acceptors denotes the oxidized, or diimine, form.
}

plasts by the addition of $p$-phenylenediimines or lipophilic $p$-benzoquinones $(2,3)$. DBMIB has, therefore, been extensively used to inhibit photosystem I electron transport and to produce conditions for the selective assay of photosystem II electron transport activity. Recently, thiol compounds have been shown to reverse DBMIB inhibition of electron transport between the two photosystems, presumably by reacting directly with DBMIB to produce a noninhibitory product (4).

DBMIB has also been shown to inhibit both PS II- and PS I-supported cyclic electron transport reactions. Although PS I cyclic reactions supported by catalysts such as DAD or phenazinemethosulfate are insensitive to DBMIB $(3,5)$, cyclic reactions catalyzed by ferredoxin or sulfonated quinones are strongly inhibited at DBMIB concentrations of from 1 to $5 \mu \mathrm{M}(5,6)$. PS II cyclic electron electron transport in $\mathrm{KCN} / \mathrm{Hg} / \mathrm{NH}_{2} \mathrm{OH}$-inhibited chloroplasts is also sensitive to DBMIB, although the extent of inhibition in this reaction system 
depends upon the type of catalyst used to promote the cycle. Activity catalyzed by an electron donor (TMPD) is more than 95\% inhibited by $12 \mu \mathrm{M}$ DBMIB, but the activity supported by the proton/electron donor PD is only diminished $60 \%$ by the same concentration of inhibitor. The sensitivity of such cyclic reactions to inhibition by DBMIB has been taken to indicate that plastoquinone participates in the coupling of cyclic electron transport to photophosphorylation in these systems (5-7).

DBMIB can also support electron transport by itself in untreated and in KCNtreated chloroplasts $(3,8)$. Under these conditions, DBMIB acts as a class III electron acceptor by catalyzing the transfer of electrons from PS II to ferricyanide, or to oxygen in a Mehler reaction (8). The concentrations of DBMIB $(10 \mu \mathrm{M})$ required for optimal activity in electron transport reactions greatly exceed, however, the concentrations $(1 \mu \mathrm{M})$ required for maximal inhibition of noncyclic electron transport from water to either ferricyanide or methylviologen.

In this communication, we present the results of experiments demonstrating the existence of a second site of DBMIB inhibition in the photosynthetic electron transport chain of spinach chloroplasts. This second site of DBMIB inhibition requires a high concentration of DBMIB $(10 \mu \mathrm{M})$ and blocks PS II electron transport reactions supported by $p$-phenylenediimines or $p$-benzoquinones. The inhibited rate of electron transport approaches the rate of electron transport supported by DBMIB alone, which indicates that DBMIB is itself catalyzing electron transport under these conditions. Attempts to reverse this inhibition with thiols have revealed major differences between this inhibition site and the site of DBMIB inhibition associated with plastoquinone function. The times of incubation and the concentrations of thiol required to affect reversals of these inhibitions indicate that the two sites of DBMIB inhibition differ in their accessability to the thiol compounds which we have employed.

\section{MATERIALS AND METHODS}

Spinach (Spinacia oleracea L.) chloroplasts were isolated (9), subjected to $\mathrm{KCN} / \mathrm{Hg}$ treatment to;impair plastocyanin function, and stored in $0.5-\mathrm{ml}$ aliquots at $-70^{\circ} \mathrm{C}$ as described earlier (10). Flectron transport activities were assayed using thawed chloroplasts by measuring light-induced changes in $\mathrm{O}_{2}$ concentration using a Clark-type oxygen electrode (YSI) fitted to a $1.6-\mathrm{ml}$ thermostatted $\left(25^{\circ} \mathrm{C}\right)$ cuvette $(10)$. The reaction mixture contained $20 \mathrm{~mm}$ Tricine $(\mathrm{pH} 8), 62.5 \mathrm{~mm}$ $\mathrm{NaCl}$, chloroplasts $(25-30 \mu \mathrm{g} \mathrm{Chl} / \mathrm{ml})$, and the electron acceptors noted in Results.

White light $\left(10^{i} \mathrm{ergs} / \mathrm{cm}^{2} \cdot \mathrm{s}\right)$ was provided by an Oriel model 6325 light source and was passed through $5 \mathrm{~cm}$ of $0.2 \% \mathrm{CuSO}_{1}$, which served as a heat filter. In experiments where light intensities were varied, fine meshed wire screens were used as neutral density filters and the radiation incident on the thermostatted $\mathrm{O}_{2}$ electrode cuvette was measured for each experiment using a YSI model 65 radiometer.

Electron acceptors used to support PS II noncyclic electron transport were recrystallized prior to use in assays (11). DMQ, DCBQ, and DBMIB were prepared as concentrated ethanolic solutions ( $20 \mathrm{mM}$ ); the concentration of organic solvent in assay mixtures never exceeded $2 \%$. The other acceptors used in these studies (PD, DAD, DAT) were dissolved in $\mathrm{H}_{2} \mathrm{O}$ to a final concentration of $20 \mathrm{~mm}$.

Tricine, bovine serum albumin, and PD were obtained from Sigma. The remainder of the electron carriers were obtained from Eastman (DMQ, DCBQ), Aldrich (DAT), or Research Organics/Inorganics (DAD). Gramicidin was from Schwarz/Mann; DBMIB was a gift from Dr. N. E. Good.

\section{RESULTS}

At a concentration of approximately 1 $\mu \mathrm{M}$, DBMIB inhibits noncyclic electron transport between PS II and PS I, and this inhibition is subsequently reversed by addition of $p$-phenylenediimines or $p$-benzoquinones, which restore PS II-dependent noncyclic electron flow. Figure 1 shows the results of an experiment in which several electron transfer reactions were assayed in the presence of increasing concentrations of DBMIB. Electron transport from water to ferricyanide (Fig. 1A), both in the absence and presence of $5 \mu \mathrm{g}$ gramicidin, is severely inhibited at a DBMIB concentration of about $1 \mu \mathrm{M}$. Electron transport which is dependent on $250 \mu \mathrm{M} \mathrm{PD}_{\mathrm{ox}}$ (Fig. 1, $A$ and $B$ ), on the other hand, is inhibited only $10 \%$ by this DBMIB concentration in both untreated and $\mathrm{KCN} / \mathrm{Hg}$-inhibited chloroplasts. Figure 1 shows, however, that if the concentration of DBMIB is increased beyond $1 \mu \mathrm{M}$, the rate of $\mathrm{PD}_{\mathrm{ox}}$-supported electron transport is decreased to a rate 


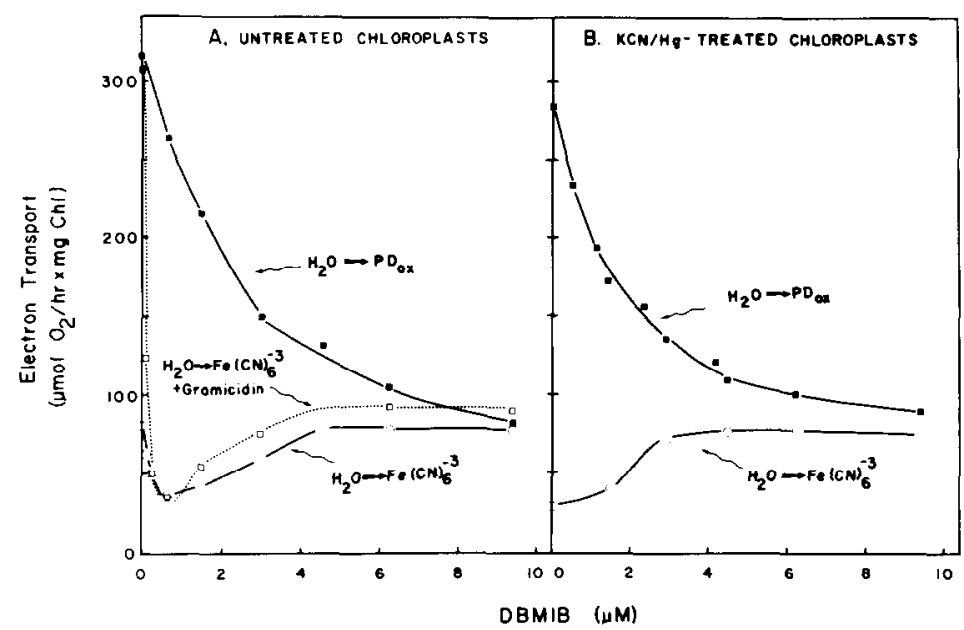

FIG. 1. Effects of DBMIB on electron transport in untreated $(A)$ and $\mathrm{KCN} / \mathrm{Hg}$-inhibited $(B)$ chloroplasts. DBMIB was added prior to chloroplast addition and all assays contained ferricyanide (2.5 $\mathrm{mM}$ ). The $\mathrm{PD}_{\mathrm{ux}}$ concentration, when present ( solid squares), was $250 \mu \mathrm{M}$. The dashed line in $A$ represents assays carried out in the presence of $5 \mu \mathrm{g}$ of gramicidin. Other conditions are as given in Materials and Methods.

which is similar to that of electron transport supported by DBMIB alone. The potency of DBMIB inhibition of electron transport mediated by $\mathrm{PD}_{\mathrm{ox}}$ is the same in both untreated and $\mathrm{KCN} / \mathrm{Hg}$-inhibited chloroplasts (Fig. 1, A and B). In the latter chloroplast preparation, PS I-supported electron transport has been suppressed prior to DBMIB addition, so that the inhibition of $\mathrm{PD}_{\mathrm{ox}}$-dependent electron transport shown here cannot be due solely to a cessation of PS I electron transport activity which requires $\mathrm{PD}_{\mathrm{ox}}$ as an electron acceptor.

The data in Table I demonstrate that inhibition of PS II electron transport activity by $9.4 \mu \mathrm{M}$ DBMIB is not unique to the catalytic function of $\mathrm{PD}_{\mathrm{ox}}$. $\mathrm{A}$ wide range of $p$-phenylenediimines and $p$-benzoquinones are sensitive to this concentration of DBMIB; the activities supported by all of these PS II acceptors are suppressed by DBMIB to residual rates of electron transport comparable to the rates supported by DBMIB alone. This finding indicates that, in each assay system, the residual activity we observe is due to the oxidation/reduction activity of DBMIB itself. Thus, the data in Fig. 1 and Table 1 support the conclusion that DBMIB imposes an inhibition upon two distinct sites in the photosynthetic electron transport chain. The first
TABLE I

INHIBITION BY DBMIB OF $p$-PHENYLENEDIIMINEAND $p$-BENZOQUINONE-SUPPORTEd EleCtron Transport in KCN/Hg-InhIBITEd Chloroplasts ${ }^{a}$

\begin{tabular}{lcc}
\hline Electron acceptor & \multicolumn{2}{c}{ Electron transport } \\
\cline { 2 - 3 } & No inhibitor & $\begin{array}{c}\text { DBMIB (9.4 } \\
\mu \mathrm{M})\end{array}$ \\
\hline None & 20.0 & 53.3 \\
DMQ & 84.5 & 55.2 \\
DCBQ $_{\text {DAD }_{\mathrm{ox}}}$ & 218 & 95.2 \\
DAT $_{\mathrm{ox}}$ & 210 & 82.0 \\
PD $_{\mathrm{ox}}$ & 253 & 86.0 \\
\hline
\end{tabular}

${ }^{a}$ Chloroplasts were incubated in the dark for $4 \mathrm{~min}$ prior to illumination, either in the absence or presence of 9.4 $\mu \mathrm{M}$ DBMIB. Following the 4-min incubation, 2.5 $\mathrm{mm}$ ferricyanide and $0.25 \mathrm{~mm}$ acceptor were added to the reaction cuvette and the assay was initiated by illumination. Other conditions were as described in Materials and Methods. Rates are expressed as micromoles of $\mathrm{O}_{2} / \mathrm{h} \cdot \mathrm{mg} \mathrm{Chl}$.

site has a high affinity for DBMIB and blocks electron transport between PS II and PS I as reported previously (1). The second site of inhibition, which has a lower affinity for DBMIB, is effectively masked by the first site unless resolved by the use of $p$-phenylenediimine or $p$-benzoquinone acceptors.

Gould and Izawa (8) have suggested that 
DBMIB, in addition to blocking the passage of electrons between PS II and I, decreases the quantum efficiency of PS II. de Kouchkovsky (12) also found that light intensity had a profound effect on the magnitude of DBMIB inhibition. A large effect on PS II quantum efficiency by increasing concentrations of DBMIB might therefore account for the low affinity site of DBMIB inhibition which we observe. To test this, we assayed the effects of DBMIB on PS II electron transport under varied light intensities. The results presented in Table II show that varying light intensities induce little or no variation in the extent of inhibition with high DBMIB concentrations. These results demonstrate that the low affinity site of DBMIB inhibition is not due simply to decreased quantum efficiency.

Reimer and Trebst (4) have shown that DBMIB inhibition of noncyclic electron transport from water to NADP is reversed by incubation of DBMIB with various thiol

\section{TABLE II}

The EFfects of Light INTENSITY ON THE INHIBITION BY DBMIB OF PD Dx $_{\text {-SUPPORTED }}$ EILECTRON Transport ${ }^{a}$

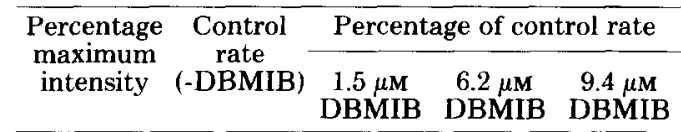

\begin{tabular}{ccccc}
\hline $\begin{array}{c}\text { Untreated } \\
\text { chloro- } \\
\text { plasts }\end{array}$ & & & & \\
100 & 503 & 66 & 34 & 32 \\
35 & 333 & 69 & 37 & 32 \\
23 & 252 & 73 & 44 & 34 \\
8 & 153 & 74 & 43 & 31 \\
KCN/Hg- & & & & \\
treated & & & & \\
chloro- & & & & \\
plasts & & & & \\
100 & 341 & 62 & 32 & 27 \\
35 & 235 & 69 & 36 & 29 \\
23 & 174 & 78 & 40 & 34 \\
8 & 117 & 70 & 39 & 33 \\
\hline
\end{tabular}

${ }^{a}$ Chloroplasts were assayed in the presence or absence of DBMIB with $2.5 \mathrm{mM}$ ferricyanide and 0.25 mM $p$-phenylenediimine. Other conditions were as described in Materials and Methods. Rates in assays minus DBMIB are expressed as micromoles of $\mathrm{O}_{2} / \mathbf{h} \cdot \mathbf{m g}$ Chl. Rates with DBMIB are expressed as a percentage of the rate minus DBMIB. The maximum intensity used was $1.1 \times 10^{6} \mathrm{ergs} / \mathrm{cm}^{2} \cdot \mathrm{s}$.

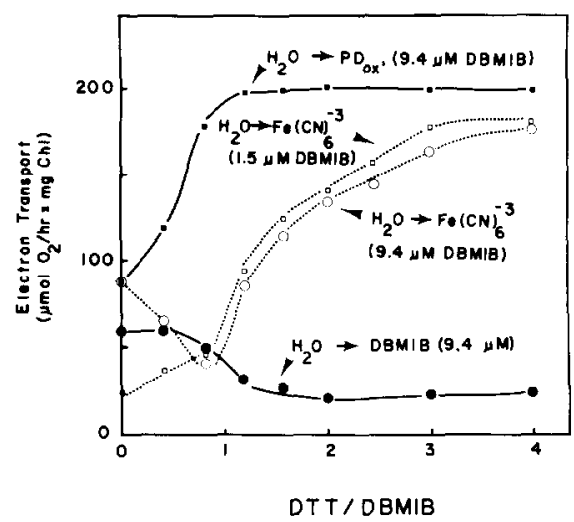

FIG. 2. Reversal of DBMIB inhibition by incubation of chloroplasts plus DBMIB with DTT. Experi mental conditions are given in the legend to Table II, except that untreated chloroplasts were used in assays represented by the dashed lines, which also included $5 \mu \mathrm{g}$ of gramicidin. Other conditions are as given in Materials and Methods.

reagents. We have tested the response of both sites of DBMIB inhibition to the thiol reagents DTT and cysteine. The results are shown in Fig. 2, where the effects of DTT were examined. Chloroplasts plus DBMIB were incubated with DTT for $2 \mathrm{~min}$ and the excess DTT was then oxidized by addition of ferricyanide ( $2.5 \mathrm{~mm}$ ) immediately before illumination. The addition of DTT to chloroplasts plus DBMIB reduced the effectiveness of DBMIB as a catalyst of PS II electron transport; increased concentrations of DTT reduce the electron transport activity supported by DBMIB in $\mathrm{KCN} / \mathrm{Hg}$ inhibited chloroplasts. DTT incubation of chloroplasts plus DBMIB reverses the inhibitory effects of DBMIB on electron flow. When electron transfer supported by ferricyanide was assayed in the presence of gramicidin, the inhibitory effects of both high $(9.4 \mu \mathrm{M})$ and low $(1.5 \mu \mathrm{M})$ concentrations of DBMIB were reversed by incubation with DTT. Increased concentrations of D'TT also reversed the DBMIB inhibition of PS II electron transport in $\mathrm{KCN} / \mathrm{Hg}$ inhibited chloroplasts. Data are shown in Fig. 2 for $\mathrm{PD}_{\mathrm{ox}}$-supported activity and in Table III for PS II reactions mediated by other quinonediimines and quinones. Thus, both the high affinity site of DBMIB inhibition, assayed using the $\mathrm{H}_{2} \mathrm{O} \rightarrow$ ferricyanide reaction, and the low affinity site, as- 
TABLE III

ReVERSAL BY DTT OF DBMIB INHIBITION OF $p$ Phenylenedilmine- and $p$-BenzoquinoneSUPPoRTED Electron Transport IN KCN/HgINHIBITED CHLOROPLASTS ${ }^{\text {" }}$

\begin{tabular}{lcc}
\hline $\begin{array}{l}\text { Electron } \\
\text { acceptor }\end{array}$ & \multicolumn{2}{c}{ Electron transport } \\
\cline { 2 - 3 } & $\begin{array}{c}\text { DTT } \\
(31 \mu \mathrm{M})\end{array}$ & $\begin{array}{c}\text { DBMIB }+ \text { DTT } \\
(9.4 \mu \mathrm{M})(31 \mu \mathrm{M})\end{array}$ \\
\hline None & 24.0 & 21.3 \\
DMQ & 84.8 & 74.5 \\
DCBQ & 201 & 178 \\
DAD $_{\mathrm{ox}}$ & 190 & 186 \\
DAT $_{\mathrm{ox}}$ & 257 & 212 \\
PD $_{b \times \mathrm{x}}$ & 246 & 205
\end{tabular}

"Chloroplasts were incubated in the dark for a total of $4 \mathrm{~min}$. DBMIB, when present, was added at the onset of incubation. DTT, when present, was added after $2 \mathrm{~min}$ of incubation had elapsed. Ferricyanide $(2.5 \mathrm{~mm})$ and an acceptor $(0.25 \mathrm{~mm})$ were added at the end of the incubation period, immediately before the assay was initiated by illumination. Control rates for these experiments are found in Table I. Other condjtions are described in Materials and Methods. Rates of $\mathrm{O}_{2}$ evolution are expressed as micromoles of $\mathrm{O}_{2} / \mathrm{h} \cdot \mathbf{m g}$ Chl.

sayed using the $\mathrm{H}_{2} \mathrm{O} \rightarrow \mathrm{PD}_{\mathrm{ox}}$ reaction, can be reversed with increased concentrations of thiol. However, as shown in Fig. 2, a higher DTT/DBMIB ratio (4:1) is required to optimally reverse the high affinity site than is required to reverse the low affinity site $(\mathrm{DTT} / \mathrm{DBMIB}=1)$. Similar results were found using cysteine. Incubation of tise chloroplasts with DTT alone did not significantly change the rates of electron transport (Table II).

The results of Fig. 2 suggested that incubation of DTT for $2 \mathrm{~min}$ with chloroplasts plus DBMIB was not sufficient to permit complete access of the thiol to the high affinity site of DBMIB inhibition. This possibility was therefore tested by varying the time interval between addition of cysteine to a cuvette containing chloroplasts and DBMIB, and the subsequent addition of ferricyanide to oxidize excess cysteine. The ability of cysteine to reverse the high affinity site of DBMIB inhibition under these conditions was assayed at two DBMIB concentrations ( 1.5 and $9.4 \mu \mathrm{M}$ ). The results, shown in Fig. 3, demonstrate a time dependency for reversal of inhibition of the noncyclic electron transport pathway from water to ferricyanide. This time-de- pendent reversal, which is optimal at about $3 \mathrm{~min}$, is partially independent of DBMIB concentration in the presence of an excess $(38 \mu \mathrm{M})$ concentration of cysteine. No time dependency greater than $10 \mathrm{~s}$ exists, however, for reversal of the low affinity site which inhibits $\mathrm{PD}_{\mathrm{ox}}$-supported activity. These findings demonstrate a fundamental difference between the two sites of DBMIB inhibition with regard to their accessability to reversal by thiol reagents.

In order to examine further the apparent difference in accessability of the two DBMIB inhibition sites, experiments were done with bovine serum albumin, which has been shown to bind DBMIB (13). The results of these experiments, presented in Fig. 4 , show that addition of bovine serum albumin to a final concentration of about 1 $\mathrm{mg} / \mathrm{ml}$ partially restores $\mathrm{PD}_{\mathrm{ox}}$-supported PS II electron transport in chloroplasts inhibited by DBMIB; addition of bovine serum albumin at this concentration does not, however, restore ferricyanide-supported electron flow, even at low DBMIB concentrations. When high concentrations of DBMIB are used to inhibit ferricyanide-

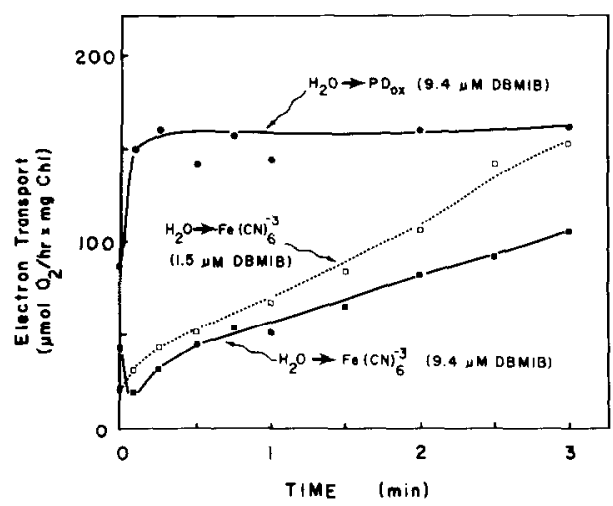

FIG. 3. Time dependency of cysteine reversal of DBMIB inhibition. Chloroplasts were incubated with DBMIB for 2 min. Cysteine was added to a final concentration of $38 \mu \mathrm{M}$. After varied periods of time, ferricyanide $(2.5 \mathrm{~mm})$ was added, oxidizing excess cysteine. The zero time point shows results where ferricyanide was added prior to cysteine addition. The $\mathrm{H}_{2} \mathrm{O}$ $\rightarrow \mathrm{PD}_{\mathrm{ox}}(250 \mu \mathrm{M})$ reaction was assayed with $\mathrm{KCN} / \mathrm{Hg}$ inhibited chloroplasts. The $\mathrm{H}_{2} \mathrm{O} \rightarrow$ ferricyanide reaction was assayed in untreated chloroplasts in the pres ence of $5 \mu \mathrm{g}$ of gramicidin. Other conditions are as given in Materials and Methods. 


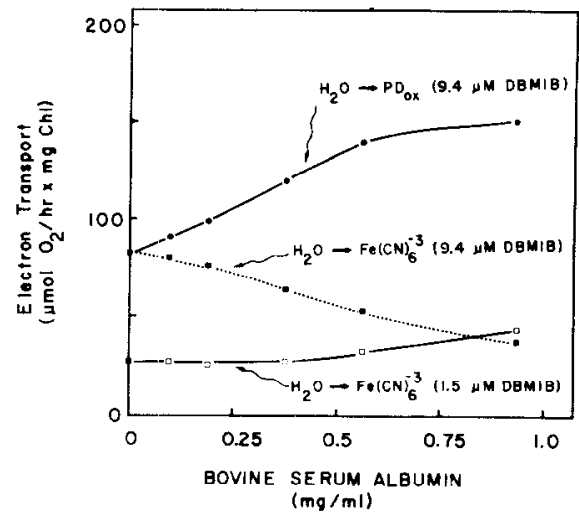

Fig. 4. Reversal of DBMIB inhibition by incubation of chloroplasts plus DRMIB with bovine serum albumin. Experimental conditions are the same as in Fig. 2, except that addition of bovine serum albumin replaced DTT addition. Only the $\mathrm{H}_{2} \mathrm{O} \rightarrow \mathrm{PD}_{\mathrm{c} \text { r }}$ reaction was assaved in $\mathrm{KCN} / \mathrm{Hg}$-inhibited chloroplasts; all other assays were carried out using untreated chloroplasts in the presence of $5 \mu \mathrm{g}$ of gramicidin.

supported electron transport, addition of bovine serum albumin actually causes a decrease in activity, probably by binding DBMIB and preventing it from participating in catalysis of electron transport activity.

\section{UISCUSSION}

The results presented here support the observations by other investigators $(2,12$, 14) that concentrations of DBMIB in excess of those required for maximal inhibition of electron transport from $\mathrm{H}_{2} \mathrm{O}$ to NADP can impose additional inhibitory effects on photosynthetic electron transport systems. Trebst and Reimer (2) demonstrated that catalytic amounts of $p$-phenylenediamine, when added to DBMIB-poisoned chloroplasts, could partially restore electron transport from $\mathrm{H}_{2} \mathrm{O}$ to NADP, but that further increases in the concentration of DBMIB caused a decrease in the amount of restoration obtained. These investigators suggested that increased concentrations of DBMIB might possibly exert these effects by influencing the fluidity of plastoquinone in the thylakoid membrane. However, since both photosystems were operative in these reactions, it would be impossible to conclusively localize the effects of increased DBMIB. de Kouchkovsky (12) has shown that PS II electron transport supported by DPIP is also influenced by high DBMIB concentrations. In this case, DBMIB appeared to disrupt the mechanism of DPIP reduction by interposing itself as an intermediate electron carrier between the membrane-bound site of DPIP reduction and the electron accepting dye.

We interpret our results (Tables I and III, Fig. 1) to indicate the existence of two distinct sites in the photosynthetic electron transport chain where DBMIB can act to interfere with noncyclic electron transport between the primary acceptor to PS II (Q) and cytochrome $f$. One of these sites has a high affinity for DBMIB and inhibits ferricyanide-supported electron flow at concentrations of about $1 \mu \mathrm{m}$. The second, lower affinity site of inhibition requires higher DBMIB concentrations $(10 \mu \mathrm{M})$ and acts to interfere with the donation of elec. trons by PS II to $p$-phenylenediimines and $p$-benzoyuinones. A method for distinguishing the two sites is also revealed by our results. The high affinity ( $1 \mu \mathrm{M}$ DBMIB) site of electron transport inhibition is reversed, under our conditions of assay, by thiol/DBMIB ratios (4:1) and times of incubation $(3 \mathrm{~min}$ ) far in excess of those required to reverse the low affinity (10 $\mu \mathrm{M}$ DBMIB) inhibition site. In addition, the two sites differ in their accessability to bovine serum albumin, which reverses part of the inhibition at the second site, but which cannot affect any reversal of inhibition at the high affinity site.

Certain conclusions concerning the low affinity site of DBMIB inhibition may be drawn from our results. Although de Kouchkovsky (12) has shown that DBMIB at high concentrations inhibits PS I activity, our use of $\mathrm{KCN} / \mathrm{Hg}$-treated chloroplasts eliminates the possibility that the DBMIB inhibitions of class III acceptor activity are due to interference by DBMIB with PS I activity. 'The data in Table II eliminate the possibility that we are observing an inhibition arising from effects of DBMIB on quantum efficiency. We can also discard uncoupling as a cause of the inhibitions we observe with $10 \mu \mathrm{M}$ DBMIB. Table I clearly demonstrates that DMQsupported PS II electron transport, which 
is uncoupler insensitive (9), is inhibited by DBMIB to the same extent as are the activities supported by $p$-phenylenediimines in a reaction which has been shown to be uncoupler sensitive (9). In addition, Gould and Izawa (8) have shown that DBMIB at concentrations far in excess of those used in the present study would support PS IIdependent ATP synthesis with phosphorylation efficiencies approaching those of PS II electron transport reactions supported by $p$-phenylenediimines. Finally, our use of $\mathrm{KCN} / \mathrm{Hg}$-inhibited chloroplasts in these studies rules out the possibility that DBMIB, at high concentrations, is by-passing the high affinity site to donate electrons to endogenous carriers which function on the oxidizing side of PS I and that DBMIB restores electron transport by a mechanism such as that suggested by Trebst and Reimer (2) for the TMPD by-pass of DBMIB inhibition at the high affinity site.

The considerations enumerated above lead us to the conclusion that the low affinity (10 $\mu \mathrm{M}$ DBMIB) site of inhibition must precede both the site of high affinity $(1 \mu \mathrm{M}$ DBMIB) inhibition as well as the site of class III acceptor reduction in the photosynthetic electron transport chain. Inhibition at the high affinity site is readily reversed by addition of $p$-phenylenediimines or $p$-benzoquinones, and the activity supported by these catalysts is in turn suppressed by the action of DBMIB at the low affinity site. It is therefore clear that DBMIB acts at the low affinity site both as an inhibitor of electron transfer to class III acceptors and as a catalyst of electron transport activity. In agreement with the earlier suggestion by de Kouchkovsky (12), we would conclude that DBMIB at high concentrations can act to accept electrons from a site close to the PS II primary acceptor. Such activity may, in turn, explain the strong fluorescence quenching activity ascribed to oxidized DBMIB (15).

Our findings contradict the results of Gould and Izawa (8). These investigators argued on the basis of inhibition studies with DCMU (3-(3,4-dichlorophenyl)-1,1-dimethylurea) and estimates of the effects of DBMIB on quantum efficiency that class III acceptors were reduced in the photosyn- thetic electron transport chain at a point closer to PS II than the site at which DBMIB was reduced. These investigators did not, however, assay the effects of DBMIB on class III acceptor activity, and this perhaps accounts for their failure to detect the low affinity site which we describe here.

At high DBMIB concentrations $(10 \mu \mathrm{M})$, $p$-phenylenediimine-supported electron transport activity cannot be distinguished from the electron transport activity catalyzed by DBMIB, and this in turn interferes with the elucidation of the nature of the inhibition at the low affinity site. The kinetics of this inhibition by DBMIB appear not to be competitive, however. At a constant, low concentration ( $1 \mu \mathrm{M})$ of DBMIB which does not, by itself, support significant rates of electron transport, varying the concentration of $\mathrm{PD}_{\text {ox }}$ in an assay system using $\mathrm{KCN} / \mathrm{Hg}$-inhibited chloroplasts produced kinetic plots which show noncompetitive inhibition by DBMIB; in addition, increasing the $\mathrm{PD}_{\mathrm{ox}}$ concentration in the presence of $10 \mu \mathrm{M}$ DBMIB does not significantly increase the overall electron transport rate (data not shown). It therefore appears likely that DBMIB, when it acts at the low affinity site, inhibits $p$-phenylenediimine reduction in a noncompetitive fashion.

Our results suggest either that the low and high affinity sites of DBMIB inhibition are topologically distinct or that differences exist between the dissociation constants for DBMIB at each site. If, for example, the high affinity site were buried within the thylakoid membrane, bovine serum albumin and thiols would have to penetrate the membrane to affect reversal of inhibition. The low affinity site, if it were located near the membrane surface, would be readily accessable to reversal agents. It is also possible that the high affinity site involves the formation of a thiol-resistant covalent bond, whereas the low affinity site does not.

\section{REFERENCES}

1. Trebst, A., Harth, E., and Draber, W. (1970) Z. Naturforsch. 25b, 1157-1159.

2. Trebst, A., AND Reimer, S. (1973) Z. Naturforsch. 28c, 710-716.

3. Izawa, S., Gould, J. M., Ort, D. R., Felker, P., ANd Good, N. E. (1973) Biochim. Biophys. Acta 
305, 119-128.

4. Reimer, S., and Trebst, A. (1976) Z. Naturforsch. 31c, 103.

5. Hauska, G., Reimer, S., and Trebst, A. (1974) Biochim. Biophys. Acta 357, 1-13.

6. Böhme, H., Reimer, S., And Trebst, A. (1971) $Z$. Naturforsch. 26b, 341-352.

7. Yocum, C. F. (1977) Plant Physiol. 60, 592-597.

8. Gould, J. M., and Izawa, S. (1973) Eur. J. Biochem. 37, 187-192.

9. Guikema, J. A., AND Yocum, C. F. (1976) Biochemistry 15, 362-367.

10. Yocum, C. F., and Guikema, J. A. (1977) Plant
Physiol. 59, 33-37.

11. Gould, J. M. (1975) Biochim. Biophys. Acta 387, 135-148.

12. DE Kouchkovsky, Y. (1974) in Proceedings of the Third International Congress on Photosynthesis (Avron, M., ed.), pp. 1081-1093, Elsevier, Amsterdam.

13. Bf,rg, S. P., and Izawa, S. (1977) Biochim. Biophys. Acta 460, 206-219.

14. Stewart, A., AND Larkum, A. W. D. (1977) Aust. J. Plant Physiol. 4, 253-261.

15. Lozier, R. H., AND Butler, W. L. (1972) FEBS Lett. 26, 161-164. 